VoL. 73 (2006) [273-283]

\title{
ON REPRESENTATIONS OF VARIANTS OF SEMIGROUPS
}

\author{
GanNa KudRyavtseva AND Victor MaltceV
}

\begin{abstract}
We construct a family of representations of an arbitrary variant $S_{a}$ of a semigroup $S$, induced by a given representation of $S$, and investigate properties of such representations and their kernels.
\end{abstract}

\section{INTRODUCTION}

Let $S$ be a semigroup and $a \in S$. Set $s * t=s a t, s, t \in S$. The semigroup $(S, *)$ is called a variant of $S$ and will be denoted by $S_{a}$. One of the motivations for the study of variants is their importance to understanding the structure of an original semigroup. In particular, the notion of a regularity-preserving element, which generalises the notion of an invertible element, is defined using variants, see $[2,3,5]$. Other situations where variants naturally appear and work are discussed in [5].

Let : $: M \times S \rightarrow M$ or just $(S, M)$ be a representation of a semigroup $S$ by transformations of a set $M$. It defines a homomorphism from $S$ to the full transformation semigroup $\mathcal{T}(M)$; we denote the image of $(m, s)$ under the function - by $m \cdot s$. For $a \in S$ and a decomposition $a=\beta \alpha, \alpha, \beta \in S^{1}$, we introduce a map $*: M \times S_{a} \rightarrow M$ or just $\left(S_{a}, M ; \alpha, \beta\right)$, defined by

$$
m * s=m \cdot(\alpha s \beta) \text { for all } s \in S, m \in M .
$$

Since

$$
\begin{aligned}
m *(s * t)=m *(s a t)= & m \cdot(\alpha s \beta \alpha t \beta) \\
& =(m \cdot(\alpha s \beta)) \cdot(\alpha t \beta)=(m * s) * t \text { for all } s, t \in S, m \in M,
\end{aligned}
$$

it follows that $\left(S_{a}, M ; \alpha, \beta\right)$ is a representation of $S_{a}$ on $M$.

The kernel $\rho$ of a representation of $S$ naturally leads to the consideration of the family of congruences $\left\{\rho_{b, c}: b, c \in S^{1}, c b=a\right\}$ on $S_{a}$, which are the kernels of the corresponding representations of $S_{a}$. In the case when $\iota$ is the identity relation on $S$, $\iota_{a, 1}$ and $\iota_{1, a}$ coincide with the congruences $l$ and $\mathrm{r}$ on $S_{a}$ respectively, which were first introduced by Symons (see [8]) for the generalised transformation semigroups and many

Received 22nd November, 2005

Copyright Clearance Centre, Inc. Serial-fee code: 0004-9727/06 \$A2.00+0.00. 
properties of which resemble the corresponding properties of the Green's relations $\mathcal{L}$ and $\mathcal{R}$.

The present paper is devoted to the study of the connections between a given representation $(S, M)$ of $S$ on $M$ and the representations $\left(S_{a}, M ; \alpha, \beta\right)$; and also to the study of the properties of congruences $\left\{\rho_{b, c}: b, c \in S^{1}, c b=a\right\}$. In Section 2 we prove that for a regular semigroup $S$, which does not contain the bicyclic semigroup, a faithful representation of $S_{a}$ coincides with certain $\left(S_{a}, M ; \alpha, \beta\right)$ if and only if $\alpha$ and $\beta$ are left and right cancellable in $S$ respectively (Theorems 1,2 and Proposition 1). We also show that for the bicyclic semigroup these conditions are not equivalent. The next result is Theorem 4, claiming that for a regular semigroup $S$ each representation * : $M \times S_{a} \rightarrow M$ of any variant of $S$ is induced by some $: M \times S \rightarrow M$ such that either $m * s=m \cdot(a s)$ for all $m \in M$ or $m * s=m \cdot(s a)$ for all $m \in M$, if and only if it is either a left or a right group, giving a new characterisation of the class of left (right) groups. In Section 3 we investigate the properties of the family of congruences $\left\{\rho_{b, c}: b, c \in S^{1}, c b=a\right\}$. In particular, in Theorem 6 we prove that in the case when $c, b$ are regular elements of $S$ the map sending $\rho$ to $\rho_{b, c}$ is a homomorphism from the lattice of congruences of $S$ to the lattice of congruences of $S_{c b}$. Without the requirement of regularity of $b, c$ this map always preserves the meet, while may not preserve the join, the corresponding example is provided. Finally, the aim of Section 4 is, given $a \in S^{1}$ and a congruence $\rho$ on $S$, to define a congruence $\rho_{a}$ which naturally generalises the congruence $\mathrm{d}$ on $S_{a}$, introduced in $[8]$ and studied afterwards in $[3,6]$.

\section{CONNECTIONS BETWEEN REPRESENTATIONS}

Let $S$ be a semigroup. An element $u \in S^{1}$ will be called left [right] cancellable if $u s=u t[s u=t u]$ implies $s=t$ for all $s, t \in S$.

Fix $a \in S$ and a decomposition $a=\beta \alpha, \alpha, \beta \in S^{1}$.

LEMMA 1. The implication $\alpha s \beta=\alpha t \beta \Rightarrow s=t$ for all $s, t \in S$ holds if and only if $\alpha$ is left cancellable and $\beta$ is right cancellable simultaneously.

Proof: The proof is straightforward.

A representation : : $M \times S \rightarrow M$ is said to be faithful if the corresponding homomorphism from $S$ to $\mathcal{T}(M)$ is injective. That is, : :M $M \rightarrow M$ is faithful if and only if

$$
(\forall m \in M)[m \cdot s=m \cdot t] \Rightarrow[s=t] \text { for all } s, t \in S \text {. }
$$

Lemma 2. Suppose that $\left(S_{a}, M ; \alpha, \beta\right)$ is faithful. Then so is $(S, M)$.

Proof: Indeed, by the definition we have

$$
(\forall m \in M)[m \cdot(\alpha s \beta)=m \cdot(\alpha t \beta)] \Rightarrow[s=t] \text { for all } s, t \in S
$$


Take $s, t \in S$. If now $m \cdot s=m \cdot t$ for all $m \in M$, then

$$
(m \cdot \alpha) \cdot(s \beta)=(m \cdot \alpha) \cdot(t \beta) \text { for all } m \in M,
$$

and so $s=t$.

THEOREM 1. Let $(S, M)$ be a representation of a semigroup $S$ on a set $M$ and $a \in S$. Take $\alpha, \beta \in S^{1}$ such that $\beta \alpha=a$. Then the following conditions are equivalent:

1. $\left(S_{a}, M ; \alpha, \beta\right)$ is faithful;

2. $(S, M)$ is faithful and $\alpha, \beta$ are left and right cancellable respectively.

Proof: That 1 implies 2 is due to Lemmas 1 and 2. The opposite implication is a consequence of Lemma 1.

Recall that an element $u$ of a semigroup $S$ is said to be a mididentity in $S$ if sut $=s t$ for all $s, t \in S$. For the case when $S$ is regular, we obtain the following theorem.

THEOREM 2. Let $S$ be a regular semigroup and $a=\beta \alpha \in S, \alpha, \beta \in S^{1}$. Suppose that there exist $\alpha^{*}$ and $\beta^{*}$ of $S^{1}$, inverses of $\alpha$ and $\beta$ in $S^{1}$ respectively, such that $\beta^{*} \beta \alpha \alpha^{*}$ is a mididentity in $S$. Let also *: $M \times S_{a} \rightarrow M$ be a faithful representation of $S_{a}$ on $M$. Then the following conditions are equivalent:

1. there is a representation $(S, M)$ such that $\left(S_{a}, M\right)$ coincides with $\left(S_{a}, M ; \alpha, \beta\right)$;

2. $\alpha$ and $\beta$ are left and right cancellable respectively.

Proof: Due to Theorem 1 we have that 1 implies 2.

Now let $\alpha$ and $\beta$ be left and right cancellable respectively. Let us prove that a function $\cdot: M \times S \rightarrow M$, given by

$$
m \cdot s=m *\left(\alpha^{*} s \beta^{*}\right) \text { for all } s \in S, m \in M,
$$

defines a representation $(S, M)$ of $S$ such that $\left(S_{a}, M\right)$ coincides with $\left(S_{a}, M ; \alpha, \beta\right)$.

Since $\beta^{*} \beta \alpha \alpha^{*}$ is a mididentity in $S$, we have

$$
\begin{aligned}
(m \cdot s) \cdot t=\left(m *\left(\alpha^{*} s \beta^{*}\right)\right) *\left(\alpha^{*} t \beta^{*}\right) & =m *\left(\alpha^{*} s \beta^{*} \cdot \beta \alpha \cdot \alpha^{*} t \beta^{*}\right) \\
& =m *\left(\alpha^{*} s t \beta^{*}\right)=m \cdot(s t) \text { for all } s, t \in S, m \in M .
\end{aligned}
$$

Thus $(S, M)$ is indeed a representation.

Note that the equality $\alpha \alpha^{*} \alpha s=\alpha s$ implies $\alpha^{*} \alpha s=s$ for all $s \in S$. Analogously, $s \beta \beta^{*}=s$ for all $s \in S$. Then we have

$$
m \cdot(\alpha s \beta)=m *\left(\alpha^{*} \alpha s \beta \beta^{*}\right)=m * s \text { for all } s \in S, m \in M .
$$

The latter means that $\left(S_{a}, M\right)$ coincides with $\left(S_{a}, M ; \alpha, \beta\right)$. That is, the function - defines the required representation $(S, M)$.

The following example shows that the condition of Theorem 2 that there are $\alpha^{*}$ and $\beta^{*}$ in $S^{1}$, such that $\beta^{*} \beta \alpha \alpha^{*}$ is a mididentity in $S$, is essential. 
EXAmple 1. Let $\mathcal{B}=\langle a, b \mid b a=1\rangle$ be the bicyclic semigroup. Consider the Cayley representation of $\mathcal{B}$ :

$$
m * s=m s \text { for all } m, s \in \mathcal{B} .
$$

Then there is no faithful representation $\circ: \mathcal{B} \times \mathcal{B} \rightarrow \mathcal{B}$ such that

$$
m \circ(a s b)=m * s=m s \text { for all } m, s \in \mathcal{B} .
$$

Since $\mathcal{B}$ is an inverse semigroup with the identity element, 1 , its Cayley representation is faithful. That $b a=1$ implies $\mathcal{B}_{b a}=B$. Note that $b^{-1} b a a^{-1}=a \cdot b a \cdot b=a b$ is not a mididentity in $\mathcal{B}$, as $1 \cdot a b \cdot 1=a b \neq 1$.

Assume that there is a faithful representation $\circ: \mathcal{B} \times \mathcal{B} \rightarrow \mathcal{B}$ such that

$$
m \circ(a s b)=m * s=m s \text { for all } m, s \in \mathcal{B} .
$$

Then $m \circ(a b)=m * 1=m$ for all $m \in \mathcal{B}$. So we have

$$
\begin{aligned}
& m \circ a=(m \circ a) \circ(a b)=m \circ\left(a^{2} b\right)=m * a=m a \text { and } \\
& m \circ b=(m \circ(a b)) \circ b=m \circ\left(a b^{2}\right)=m * b=m b \text { for all } m \in \mathcal{B} .
\end{aligned}
$$

But this leads to a contradiction:

$$
1=1 \circ(a b)=(1 \circ a) \circ b=a \circ b=a b .
$$

Let us recall (see [1, Corollary 1.32]) that every homomorphic image of $B$ is either a cyclic group or isomorphic to $\mathcal{B}$. Using this fact, we are going to prove that in the case when $S$ is a regular semigroup, which does not contain a subsemigroup, isomorphic to $\mathcal{B}$, one obtains that the conditions 1 and 2 of Theorem 2 are equivalent.

PROPOS ITION 1. Let $S$ be a regular semigroup, which does not contain a subsemigroup isomorphic to $\mathcal{B}$. Let $a=\beta \alpha \in S, \alpha, \beta \in S^{1}$. Let also *: $M \times S_{a} \rightarrow M$ be a faithful representation of $S_{a}$ on $M$. Then the conditions 1 and 2 of Theorem 2 are equivalent.

ProOF: We have only to prove that 2 implies 1.

Let now $\alpha$ and $\beta$ be left and right cancellable respectively. Take $\alpha^{*}$ and $\beta^{*}$, inverses of $\alpha$ and $\beta$ in $S^{1}$ respectively. Then the equality $\alpha \alpha^{*} \alpha s=\alpha s$ implies $\alpha^{*} \alpha s=s$ for all $s \in S$. Also $s \beta \beta^{*} \beta=s \beta$ implies $s \beta \beta^{*}=s$ for all $s \in S$. It follows that $\alpha^{*} \alpha$ and $\beta \beta^{*}$ are left and right identity elements of $S^{1}$ respectively. Consider three possible cases.

CASE 1. Let $\alpha, \beta \in S$. Then $\alpha^{*} \alpha$ and $\beta \beta^{*}$ are both in $S$. It follows that $S$ has the identity $1=\alpha^{*} \alpha=\beta \beta^{*}$. Assume that $\alpha \alpha^{*} \neq 1$. Then $\left\langle\alpha, \alpha^{*}\right\rangle$ is a homomorphic image of $\mathcal{B}=\langle a, b \mid b a=1\rangle$ under a map, which sends $a$ to $\alpha$ and $b$ to $\alpha^{*}$. Since $\left\langle\alpha, \alpha^{*}\right\rangle$ is not isomorphic to a cyclic group, then $\left\langle\alpha, \alpha^{*}\right\rangle$ is a subsemigroup, isomorphic to $\mathcal{B}$. We get a 
contradiction. Thus $\alpha \alpha^{*}=1$. Analogously, one shows that $\beta^{*} \beta=1$. Then $\beta^{*} \beta \alpha \alpha^{*}$ is a mididentity in $S$. Now due to Theorem 2, we have that 1 holds.

CASE 2. Let $\beta \notin S$ and $\alpha=a \in S$. Arguments are similar to those of Case 1. Then $\alpha \alpha^{*}=\alpha^{*} \alpha$ is again a mididentity in $S$ and to prove that 1 holds, it remains to use Theorem 2.

CASE 3. Let $\alpha \notin S$ and $\beta=a \in S$. Analogously to Case $2, \beta^{*} \beta=\beta \beta^{*}$ is a mididentity in $S$. Thus, according to Theorem 2, 1 holds.

Recall (see [1]) that a right [left] group is a semigroup which is right [left] simple and left [right] cancellative. We shall need the following known fact.

Theorem 3. ([1, Theorem 1.27].) The following conditions for a semigroup $S$ are equivalent:

1. $S$ is a left [right] group;

2. $S$ is right [left] simple and contains an idempotent;

3. $S$ is a direct product $G \times U$ of a group $G$ and a left [right] zero semigroup $U$.

Among all the decompositions $a=\beta \alpha, \alpha, \beta \in S^{1}$, two are rather special, namely $a=a \cdot 1$ and $a=1 \cdot a$. The main result of the following theorem is the characterisation of semigroups $S$ such that for all $a \in S$ and for each representation *: $M \times S_{a} \rightarrow M$ there exists a representation $: M \times S \rightarrow M$ such that either $m * s=m \cdot(a s)$ for all $m \in M$ or $m * s=m \cdot(s a)$ for all $m \in M$.

THEOREM 4. Let $S$ be a regular semigroup with the set of idempotents $E$. Then the following conditions are equivalent:

1. for all $a \in S$ and for each representation *: $M \times S_{a} \rightarrow M$ there exists a representation : $M \times S \rightarrow M$ such that either $m * s=m \cdot(a s)$ for all $m \in M$ or $m * s=m \cdot(s a)$ for all $m \in M$;

2. for all $e \in E$ there are a faithful representation * : $M \times S_{e} \rightarrow M$ and a representation : $M \times S \rightarrow M$ such that either $m * s=m \cdot(e s)$ for all $m \in M$ or $m * s=m \cdot(s e)$ for all $m \in M$;

3. every element of $E$ is either a left or a right identity in $S$;

4. $S$ is either a left or a right group.

Proof: Obviously, 1 implies 2.

Assume that 2 holds. Take an idempotent $e \in E$. The first case is that there are a set $M$, a faithful representation *: $M \times S_{e} \rightarrow M$ and a representation $\cdot: M \times S \rightarrow M$ such that $m * s=m \cdot(e s)$ for all $m \in M$. Then $m * s=m *(e s)$ for all $m \in M$ and $s \in S$ and so $s=e s$ for all $s \in S$. In the second case we obtain $s=s e$ for all $s \in S$. Thus, 2 implies 3 . 
Let us prove now that 3 implies 4 . Note that $E$ is nonempty as $S$ is regular. If $S$ contains both left and right identities then it contains the identity 1 , thus each idempotent coincides with 1 . Hence, $S$ is a regular semigroup with the unique idempotent 1 . It follows now from [7, Lemma I1.2.10] that $S$ is a group and so also a left group. If every idempotent $e \in E$ is a left [right] unit in $S$, then

$$
s S^{1}=s S=s s^{\prime} S=S\left[S^{1} s=S s=S s^{\prime} s=S\right]
$$

for all $s \in S$ and for each $s^{\prime}$, inverse of $s$, and so $S$ is right [left] simple. But due to Theorem 3 we obtain now that $S$ is a right [left] group.

Finally, assume that $S$ is, for instance, a left group. Then by Theorem 3 there are a group $G$ and a left zero semigroup $U$ such that $S=G \times U$. Take $(g, u) \in S$ and a representation * : $M \times S_{(g, u)} \rightarrow M$. Put $m \cdot(h, v)=m *\left(h g^{-1}, v\right)$ for all $(h, v) \in S$. Then $m \cdot((h, v)(g, u))=m *(h, v)$ for all $(h, v) \in S$. We are left to prove that $\cdot$ is a representation of $S$. Indeed, we have

$$
\begin{aligned}
m \cdot\left(h_{1} h_{2}, v_{1} v_{2}\right)=m * & \left(h_{1} h_{2} g^{-1}, v_{1} v_{2}\right)=m *\left(\left(h_{1} g^{-1}, v_{1}\right) *\left(h_{2} g^{-1}, v_{2}\right)\right) \\
& =\left(m \cdot\left(h_{1}, v_{1}\right)\right) \cdot\left(h_{2}, v_{2}\right) \text { for all }\left(h_{1}, v_{1}\right),\left(h_{2}, v_{2}\right) \in S, m \in M
\end{aligned}
$$

This completes the proof.

Let $S$ be a semigroup and $\cdot: M \times S \rightarrow M$ a representation of $S$. Denote by $M \cdot \alpha$ the set $\{m \cdot \alpha: m \in M\}$ and by $m \cdot S$ the set $\{m \cdot s: s \in S\}$. In the case when $S \neq S^{1}$ set $M \cdot 1=M$. A representation $(S, M)$ is said to be cyclic (see [1]) if there is a generating element $m \in M$ for $(S, M)$, that is, $m \cdot S=M$.

Proposition 2. Let $S$ be an arbitrary semigroup and $a \in S, a=\beta \alpha, \alpha, \beta$ $\in S^{\mathbf{1}}$. Let $(S, M)$ be a representation of $S$ on $M$. The following conditions are equivalent:

1. $\left(S_{a}, M ; \alpha, \beta\right)$ is cyclic;

2. $(S, M)$ is cyclic, $M \cdot \beta=M$ and $M \cdot \alpha$ contains a generating element for $(S, M)$.

Proof: Suppose that 1 holds. It follows immediately from the definition of cyclic representations that $(S, M)$ is cyclic. If $m \in M$ is a generating element for $\left(S_{a}, M ; \alpha, \beta\right)$ then $m \cdot(\alpha S \beta)=M$ which implies $M \cdot \beta=M$. Since

$$
(m \cdot \alpha) \cdot S \supseteq m \cdot(\alpha S \beta)=M,
$$

we have that $M \cdot \alpha$ contains a generating element for $(S, M)$.

Suppose now that 2 holds. Let $m \in M \cdot \alpha$ be a generating element for $(S, M)$ such that $m=m_{0} \alpha$ for some $m_{0} \in M$. Let us prove that $m_{0}$ is a generating element for $\left(S_{a}, M ; \alpha, \beta\right)$. Indeed, take $m_{1} \in M$. Then there exists $\bar{m} \in M$ such that $m_{1}=\bar{m} \cdot \beta$. Also there is $s \in S$ such that $\bar{m}=\left(m_{0} \alpha\right) \cdot s$. Then we obtain $m_{0} * s=m_{0} \cdot(\alpha s \beta)$ $=\left(\left(m_{0} \alpha\right) \cdot s\right) \cdot \beta=m_{1}$. Thus $m_{0} * S=M$. 


\section{Congruences $\rho_{b, c}$}

Let $\rho$ be a congruence on a semigroup $S$ and $a \in S, a=c b, b, c \in S^{1}$. Define a relation $\rho_{b, c}$ on $S_{a}$ as follows:

$$
(s, t) \in \rho_{b, c} \text { if and only if }(b s c, b t c) \in \rho \text { for all } s, t \in S .
$$

It is straightforward that $\rho_{b, c}$ is a congruence on $S_{a}$. If now we have a representation $\cdot: M \times S \rightarrow M$ of $S$ on $M$, then the congruence $\nu$, related to it, is given by

$$
(s, t) \in \nu \text { if and only if } m \cdot s=m \cdot t \text { for all } m \in M \text {. }
$$

Then one can easily see that the congruence on $S_{a}$, related to the representation $\left(S_{a}, M ; b, c\right)$, coincides with $\nu_{b, c}$.

Denote by $\operatorname{Cong}(S)$ the set of all congruences on a semigroup $S$. Set $\rho_{1,1}=\rho$ for all $\rho \in \operatorname{Cong}(S)$. In the case when $S \neq S^{1}$, set also $S_{1}=S$.

Proposition 3. Let $S$ be a semigroup, $b, c \in S^{1}, \rho \in \operatorname{Cong}(S)$. Then

$$
S_{c b} / \rho_{b, c} \cong b S c / \rho \cap(b S c \times b S c) \text {. }
$$

Proof: Define a map $\varphi: S_{c b} \rightarrow b S c / \rho \cap(b S c \times b S c)$ as follows:

$$
\varphi(x)=(b x c) \rho \text { for all } x \in S .
$$

One can easily show that $\varphi$ is an onto homomorphism and $\varphi \circ \varphi^{-1}=\rho_{b, c}$. These two facts complete the proof.

Let $S \neq S^{1}$ and $\rho \in \operatorname{Cong}(S)$. Then we identify (1) $\rho$ with the identity element of $(S / \rho)^{1}$.

THEOREM 5. Let $S$ be a semigroup, $b, c, b_{1}, c_{1} \in S^{1}, \rho, \sigma \in \operatorname{Cong}(S)$. Then

1. if $b \rho$ and $b_{1} \rho$ are $\mathcal{L}$-related, $c \rho$ and $c_{1} \rho$ are $\mathcal{R}$-related in $(S / \rho)^{1}$, then $\rho_{b, c}$ $=p_{b_{1}, c_{1}}$;

2. $\rho \subseteq \rho_{b, c} ;$

3. $\rho_{b, c}=\rho$ if and only if $b \rho$ and $c \rho$ are left and right cancellable in $(S / \rho)^{1}$ respectively;

4. if $\rho \subseteq \sigma$ then $\rho_{b, c} \subseteq \sigma_{b, c}$. If $b \sigma$ and $c \sigma$ are left and right cancellable in $(S / \sigma)^{1}$ then $\rho \subseteq \sigma$ if and only if $\rho_{b, c} \subseteq \sigma_{b, c}$.

PROof: Statements 1 and 2 follow immediately from the definition of $\rho_{b, c}$. Statement 3 follows from Lemma 1 and the fact that $\rho_{b, c}=\rho$ is equivalent to the implication

$$
(b \rho) x(c \rho)=(b \rho) y(c \rho) \Rightarrow[x=y] \text { for all } x, y \in S / \rho .
$$

Finally, let us prove 4. If $\rho \subseteq \sigma$ then $(x, y) \in \rho_{b, c}$ implies $(b x c, b y c) \in \rho \subseteq \sigma$ which, in turn, implies $(x, y) \in \sigma_{b, c}$. Thus, if $\rho \subseteq \sigma$, then $\rho_{b, c} \subseteq \sigma_{b, c}$. 
Suppose now that $b \sigma$ and $c \sigma$ are left and right cancellable in $(S / \sigma)^{1}$ and $\rho_{b, c}$ $\subseteq \sigma_{b, c}$. Take $(x, y) \in \rho$. Then $(b x c, b y c) \in \rho$ which implies $(x, y) \in \rho_{b, c} \subseteq \sigma_{b, c}$ or just $(b \sigma)(x \sigma)(c \sigma)=(b \sigma)(y \sigma)(c \sigma)$, we have $x \sigma=y \sigma$. Thus, we obtain that $\rho \subseteq \sigma$. This completes the proof.

The converse statement of 1 of Theorem 5 is not true in general as the following easy example shows.

EXAMPLE 2. Consider a semilattice $E=\{a, b\}$, where $a \leqslant b$. Let $\rho$ be the identity relation on $E$. Then $\rho_{a, a}=\rho_{a, b}$ but $(a, b) \notin \mathcal{R}$.

Set $\rho_{a}^{r}=\rho_{1, a}$ and $\rho_{a}^{l}=\rho_{a, 1}$ for all congruences $\rho$ on a semigroup $S$ and $a \in S^{1}$. The following proposition shows that the converse statement of 1 of Theorem 5 is true in the case when $b=b_{1}=1\left[c=c_{1}=1\right]$ and $S / \rho$ is inverse.

Proposition 4. Let $S$ be a semigroup, $b, c \in S^{1}, \rho \in \operatorname{Cong}(S)$. Suppose that $S / \rho$ is inverse. Then $\rho_{b}^{r}=\rho_{c}^{r}\left[\rho_{b}^{l}=\rho_{c}^{l}\right]$ if and only if $b \rho$ and $c \rho$ are $\mathcal{R}$-related [L-related] in $(S / \rho)^{1}$.

Proof: The sufficiency follows from 1 of Theorem 5. Let now assume that $\rho_{b}^{r}=\rho_{c}^{r}$. Set $b \rho=u$ and $c \rho=v$. Then we have $x u=y u$ if and only if $x v=y v$ for all $x, y \in S / \rho$. In particular, $x u=x u u^{-1} u$ gives us $x v=x u u^{-1} v$ for all $x \in S / \rho$. Hence, $v=v v^{-1} v$ $=v v^{-1} u u^{-1} v=u u^{-1} v$, thus $v v^{-1}=u u^{-1} v v^{-1}$. Analogously, $u u^{-1}=v v^{-1} u u^{-1}$. Thus, $v v^{-1}=u u^{-1}$ which is well-known to be equivalent to $u \mathcal{R} v$ (see [4]).

Take $b, c \in S^{1}$. Set a map $\varphi_{b, c}: \operatorname{Cong}(S) \rightarrow \operatorname{Cong}\left(S_{c b}\right)$ as follows:

$$
\varphi_{b, c}(\rho)=\rho_{b, c} \text { for all } \rho \in \operatorname{Cong}(S) \text {. }
$$

It is well-known that if one has $\rho, \sigma \in \operatorname{Cong}(S)$ then $\rho \vee \sigma$ coincides with the transitive closure $(\rho \cup \sigma)^{t}$. Denote by $\mathcal{S L}(S)$ the lower semilattice (Cong $\left.(S) ; \subseteq, \cap\right)$ of congruences on $S$ and by $\mathcal{L}(S)$ the lattice $(\operatorname{Cong}(S) ; \subseteq, \cap, \vee)$ of congruences on $S$.

THEOREM 6. Let $S$ be a semigroup and $b, c \in S^{1}$. Then

1. $\varphi_{b, c}$ is a homomorphism from $\mathcal{S L}(S)$ to $\mathcal{S L}\left(S_{c b}\right)$;

2. $\rho_{b, c} \vee \sigma_{b, c} \subseteq(\rho \vee \sigma)_{b, c}$ for all $\rho, \sigma \in \operatorname{Cong}(S)$;

3. if $b$ and $c$ are regular in $S^{1}$ then $\varphi_{b, c}$ is a homomorphism from $\mathcal{L}(S)$ to $\mathcal{L}\left(S_{c b}\right)$

4. if $b S c=S$ then $\varphi_{b, c}$ is injective homomorphism from $\mathcal{L}(S)$ to $\mathcal{L}\left(S_{c b}\right)$.

Proof: Take $\rho, \sigma \in \operatorname{Cong}(S)$. Then $(x, y) \in \rho_{b, c} \cap \sigma_{b, c}$ if and only if $(b x c, b y c) \in \rho \cap \sigma$, which is, in turn, equivalent to $(x, y) \in(\rho \cap \sigma)_{b, c}$ for all $x, y \in S$. Thus, $\rho_{b, c} \cap \sigma_{b, c}$ $=(\rho \cap \sigma)_{b, c}$, which completes the proof of 1 .

To prove 2, we note that due to $\rho_{b, c} \subseteq(\rho \vee \sigma)_{b, c}$ and $\sigma_{b, c} \subseteq(\rho \vee \sigma)_{b, c}$, we have that $\rho_{b, c} \vee \sigma_{b, c} \subseteq(\rho \vee \sigma)_{b, c}$. 
Let now $b$ and $c$ be regular in $S^{1}$ and $b^{\prime}, c^{\prime}$ be certain inverses of $b$ and $c$ in $S^{1}$ respectively. To prove 3 , in view of what has already been done, we are left to show that $(\rho \vee \sigma)_{b, c} \subseteq \rho_{b, c} \vee \sigma_{b, c}$. Suppose that $(x, y) \in(\rho \vee \sigma)_{b, c}$. Then $(b x c, b y c) \in \rho \vee \sigma$ and so there are $p_{1}, \ldots, p_{m} \in S$ such that

$$
\left(b x c, p_{1}\right) \in \rho \cup \sigma, \ldots,\left(p_{i}, p_{i+1}\right) \in \rho \cup \sigma, \ldots,\left(p_{m}, b y c\right) \in \rho \cup \sigma .
$$

Clearly, $\rho \cup \sigma$ is left and right compatible, and therefore

$$
\begin{aligned}
\left(b x c, b \cdot b^{\prime} p_{1} c^{\prime} \cdot c\right) \in & \rho \cup \sigma, \ldots, \\
& \left(b \cdot b^{\prime} p_{i} c^{\prime} \cdot c, b \cdot b^{\prime} p_{i+1} c^{\prime} \cdot c\right) \in \rho \cup \sigma, \ldots,\left(b \cdot b^{\prime} p_{m} c^{\prime} \cdot c, b y c\right) \in \rho \cup \sigma,
\end{aligned}
$$

which yields

$$
\left(x, b^{\prime} p_{1} c^{\prime}\right) \in \rho_{b, c} \cup \sigma_{b, c}, \ldots,\left(b^{\prime} p_{i} c^{\prime}, b^{\prime} p_{i+1} c^{\prime}\right) \in \rho_{b, c} \cup \sigma_{b, c}, \ldots,\left(b^{\prime} p_{m} c^{\prime}, y\right) \in \rho_{b, c} \cup \sigma_{b, c}
$$

then $(x, y) \in \rho_{b, c} \vee \sigma_{b, c}$. Thus, 3 is proved.

Finally, assume that $b S c=S$. To prove that $\varphi_{b, c}$ is a homomorphism, it is enough to show that $(\rho \vee \sigma)_{b, c} \subseteq \rho_{b, c} \vee \sigma_{b, c}$. Take $(x, y) \in(\rho \vee \sigma)_{b, c}$. Then there are $p_{1}, \ldots, p_{m} \in S$ such that (1) holds. Then due to $b S c=S$ and the fact that $(b s c, b t c) \in \rho \cup \sigma$ if and only if $(s, t) \in \rho_{b, c} \cup \sigma_{b, c}$ for all $s, t \in S$, we obtain that $(x, y) \in \rho_{b, c} \vee \sigma_{b, c}$. It remains to prove that $\varphi_{b, c}$ is injective. Suppose that $\rho_{b, c}=\sigma_{b, c}$. Take $(s, t) \in \rho$. There are $s_{1}$ and $t_{1}$ of $S$ such that $s=b s_{1} c$ and $t=b t_{1} c$. Then $\left(s_{1}, t_{1}\right) \in \rho_{b, c}$ which implies $\left(s_{1}, t_{1}\right) \in \sigma_{b, c}$, which, in turn, is equivalent to $(s, t) \in \sigma$. Thus, $\rho \subseteq \sigma$. Analogously, $\sigma \subseteq \rho$. So $\rho=\sigma$ and $\varphi_{b, c}$ is an injective homomorphism.

We note that the converse inclusion of one in 2 of Theorem 6, namely $(\rho \vee \sigma)_{b, c}$ $\subseteq \rho_{b, c} \vee \sigma_{b, c}$ for $\rho, \sigma \in \operatorname{Cong}(S)$, is not true in general as the following example illustrates.

EXAMPLE 3. Consider the free semigroup $\{a, b\}^{+}$over the alphabet $\{a, b\}$. Let $I$ be the ideal consisting of all words from $\{a, b\}^{+}$of length not less than 3. Set $S=\{a, b\}^{+} / I$ to be the Rees quotient semigroup.

Set $\rho=(b a, a b) \cup(a b, b a) \cup \iota$ and $\sigma=(a b, b b) \cup(b b, a b) \cup \iota$, where $\iota$ denotes the identity relation on $S$. It follows immediately from the construction of $S$ that $\rho, \sigma \in \operatorname{Cong}(S)$. Now we observe that $(a, b) \in(\rho \vee \sigma)_{b, 1}$. Indeed, we have $(b a, a b) \in \rho$ and $(a b, b b) \in \sigma$, thus $(b a, b b) \in \rho \vee \sigma$ which is equivalent to $(a, b) \in(\rho \vee \sigma)_{b, 1}$. But $(a, b) \notin \rho_{b, 1} \vee \sigma_{b, 1}$. Indeed, otherwise there would exist $t_{1}, \ldots, t_{n} \in S$ such that

$$
\left(b a, b t_{1}\right) \in \rho \cup \sigma, \ldots,\left(b t_{i}, b t_{i+1}\right) \in \rho \cup \sigma, \ldots,\left(b t_{n}, b b\right) \in \rho \cup \sigma .
$$

It follows from the construction of $\rho$ and $\sigma$ that $\left(b a, b t_{1}\right) \in \rho \cup \sigma$ implies $t_{1}=a$. Now inductive arguments show that $t_{i}=a$ for all possible $i$. In particular, $t_{n}=a$. Then $(b a, b b) \in \rho \cup \sigma$, and we get a contradiction. Thus, $(\rho \vee \sigma)_{b, 1} \nsubseteq \rho_{b, 1} \vee \sigma_{b, 1}$. 


\section{Congruences $\rho_{a}$}

Let now $\rho$ be a congruence on a semigroup $S$ and $a \in S^{1}$. Then we can construct a congruence $\rho_{a} \in \operatorname{Cong}\left(S_{a}\right)$ as follows:

$$
(s, t) \in \rho_{a} \text { if and only if }(a s a, a t a) \in \rho \text { for all } s, t \in S \text {. }
$$

Thus, in terms of [3], $(s, t) \in \rho_{a}$ if and only if $(s \rho, t \rho) \in \delta^{a \rho}$.

If now one has $a=c b, b, c \in S^{1}$, then $\rho_{b, c} \subseteq \rho_{a}$. The following statement shows when the opposite inclusion holds in the case when $S / \rho$ is inverse.

Proposition 5. Let $S$ be a semigroup, $b, c \in S^{1}$. Let also $\rho$ be a congruence on $S$ such that $S / \rho$ is inverse. Then $\rho_{b, c}=\rho_{c b}$ if and only if

$$
u x v=v^{-1} v u \cdot x \cdot v u u^{-1} \text { for all } x \in S / \rho,
$$

where $u=b \rho$ and $v=c \rho$.

ProOF: The condition $\rho_{b, c}=\rho_{c b}$ is equivalent to $\rho_{c b} \subseteq \rho_{b, c}$, which is, in turn, equivalent to

$$
v \cdot u x v \cdot u=v \cdot u y v \cdot u \Rightarrow u x v=u y v \text { for all } x, y \in S / \rho \text {. }
$$

Let us prove that the condition (3) is equivalent to (2).

Indeed, assume that (3) holds. Since

$$
v u \cdot x \cdot v u=v u \cdot u^{-1} v^{-1} v u \cdot x \cdot v u u^{-1} v^{-1} \cdot v u \text { for all } x \in S / \rho,
$$

we have

$$
u \cdot x \cdot v=u \cdot u^{-1} v^{-1} v u \cdot x \cdot v u u^{-1} v^{-1} \cdot v=v^{-1} v u \cdot x \cdot v u u^{-1},
$$

due to the fact that all the idempotents of an inverse semigroup pairwise commute. That is, the condition (2) holds.

Assume now that (2) holds. Suppose that $v \cdot u x v \cdot u=v \cdot u y v \cdot u$ for some $x, y \in S / \rho$. Then

$$
u x v=v^{-1} v u \cdot x \cdot v u u^{-1}=v^{-1} v u \cdot y \cdot v u u^{-1}=u y v,
$$

then we obtain (3).

Proposition 6. Let $S$ be a semigroup, $a \in S^{1}, \rho \in \operatorname{Cong}(S)$. Then

$$
S_{a} / \rho_{a} \cong a S a / \rho \cap(a S a \times a S a)
$$

Proof: Set a map $\psi: S_{a} \rightarrow a S a / \rho \cap(a S a \times a S a)$ as follows:

$$
\psi(x)=(a x a) \rho \text { for all } x \in S .
$$

It remains to note that $\psi$ is an onto homomorphism and $\psi \circ \psi^{-1}=\rho_{a}$. 


\title{
REFERENCES
}

[1] A.H. Clifford and G.B. Preston, The algebraic theory of semigroups, Mathematical Surveys 7 (American Mathematical Society, Providence, R.I., 1961, 1967).

[2] J. Hickey, 'Semigroups under a sandwich operation', Proc. Edinburgh Math. Soc. 26 (1983), 371-382.

[3] J. Hickey, 'On variants of a semigroup', Bull. Austral. Math. Soc. 34 (1986), 447-459.

[4] P. Higgins, Techniques of semigroup theory (Oxford University Press, New York, 1992).

[5] T. Khan and M. Lawson, 'Variants of regular semigroups', Semigroup Forum 62 (2001), 358-374.

[6] K. Magill, P. Misra and U. Tewari, 'Symons' d-congruence on sandwich semigroups', Czechslovak Math. J. 33 (1983), 221-236.

[7] M. Petrich, Inverse semigroups (John Wiley \& Sons, 1984).

[8] J. Symons, 'On a generalization of the transformation semigroups', J. Austral. Math. Soc. 19 (1975), 47-61.

\author{
Algebra, Department of Mathematics and Mechanics \\ Kyiv Taras Shevchenko University \\ 64 Volodymyrska St. \\ 01033 Kyiv \\ Ukraine \\ e-mail: akudr@univ.kiev.ua \\ vmaltcev@univ.kiev.ua
}

